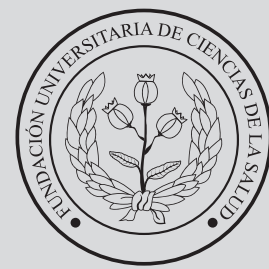

FUCS
Re

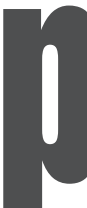

\title{
,

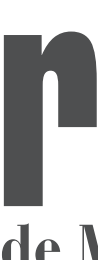

\section{Descripción estadística del COVID- 19 según el grupo etario en Colombia}

Jorge Enrique Díaz-Pinzón ${ }^{a}$

\section{Statistical analysis of COVID-19 cases according to age group in Colombia}

${ }^{a}$ Ingeniero. Magister en Gestión de la Tecnología Educativa, Especialista en Administración de la Informática Educativa. Docente de matemáticas e Investigador, Secretaría de Educación de Soacha, Cundinamarca.

\section{R E S U M E N}

Introducción: a medida que el número de casos de COVID-19 se prolonga, los servicios de salud locales corren el riesgo de ser agobiados con pacientes que requieren cuidados intensivos. Por tal motivo se realizó un estudio de investigación de los resultados de personas contagiadas, recuperadas, y fallecidas por la enfermedad y su distribución por grupo etario en Colombia. Objetivo: comparar si hay diferencias en los promedios de personas con contagio, recuperadas y fallecidas por COVID-19 entre los diferentes grupos etarios. Materiales y métodos: se utilizó la base de datos de las personas infectadas por el COVID-19 con la información acumulada entre el 6 y 10 de julio de 2020. Se realizó a partir del análisis estadístico de los datos aplicando la ANOVA, y una prueba post hoc de Duncan, se utilizó el paquete estadístico SPSS v. 25. Resultados: a partir del análisis estadístico de los datos aplicando la comparación de medias con el ANOVA, el p-valor de las pruebas, para la variable contagios, recuperados y fallecidos fue de 0,000, este es menor a $a=0.05$, de esta manera se rechaza Ho, es decir que para al menos uno de los promedios es diferente de los demás del resultado obtenido para los diferentes grupos etarios. Conclusiones: el grupo etario más vulnerable al contagio está en el rango de 30-39, en el tema de personas recuperadas se presentó en este mismo rango, y para el caso de personas fallecidas se ubicó en el rango de 70-79.

Palabras clave: COVID-19, coronavirus, grupo etario, pandemia.

(C) 2020 Fundación Universitaria de Ciencias de la Salud - FUCS. Este es un artículo Open Access bajo la licencia CC BY-NC-ND (http://creativecommons.org/licenses/by-nc-nd/4.0/).

\section{INFORMACIÓN DEL ARTÍCULO}

Historia del artículo:

Fecha recibido: julio 21 de 2020

Fecha aceptado: julio 26 de 2020
Autor para correspondencia.

Ing. Jorge Enrique Díaz Pinzón jediazp@unal.edu.co
DOI

10.31260/RepertMedCir.01217372.1089 
Introduction: as the number of COVID-19 cases continues to rise, local health services are at risk of being overwhelmed with patients requiring intensive care. For this reason, a research study was carried out on the outcomes of people infected, recovered and killed by the disease and its distribution by age groups in Colombia. Objective: to compare if there are differences in the averages of positive cases, recoveries and COVID-19-related deaths by age groups. Materials and Methods: the database including people infected with COVID-19 was used, with the information accumulated between July 6 and July 102020 . A statistical analysis of this data was made by applying the ANOVA and a Duncan post hoc multiple comparisons test, in the SPSS v.25 statistical package. Results: from the statistical analysis of said data applying the ANOVA mean comparison, it was determined that the $\mathrm{p}$ value for the transmission, recoveries and deaths variables was equal to 0.000 , which is less than $a$ $=0.05$, thus, the hypothesis (Ho) was rejected, which means that at least for one of the averages, it is different from the rest of the results obtained for the different age groups. Conclusions: the most vulnerable group to COVID-19 infection is the 30 to 39 age group. Individuals who have recovered are in this same age group. The COVID-19-related deaths have occurred among people between $70-79$ years of age.

Key words: COVID-19 - coronavirus, age group; pandemic

(C) 2020 Fundación Universitaria de Ciencias de la Salud - FUCS. This is an open access article under the CC BY-NC-ND license (http://creativecommons.org/licenses/by-nc-nd/4.0/).

\section{INTRODUCCIÓN}

El brote de COVID-19 ${ }^{1}$, la enfermedad causada por el nuevo coronavirus SARS-CoV-2, descubierto en China en diciembre de $2019^{2}$, se ha transformado en una pandemia y continúa ubicando sistemas nacionales de salud de diferentes países en niveles significativos de estrés. ${ }^{3,4,5,6}$

En la pandemia de COVID-19, la demanda de cuidados intensivos no es igual en todo el país. Los brotes epidémicos pueden tomar diferentes partes de un territorio y esto puede conducir a diversidad de la demanda tanto a través del espacio como del tiempo. Algunos hospitales logran recibir un número colosal de pacientes al comienzo de un brote, mientras que otros pueden ser solo ligeramente afectados. Esta heterogeneidad de la demanda abre la contingencia de equilibrar la carga de ingresos de pacientes como esa demanda fenomenal se redirige a los lugares que tienen capacidad libre. ${ }^{7}$

En Colombia, se detectó el primer caso por COVID-19 el 6 de marzo de 2020, estudios de investigación en Colombia como el de Díaz ${ }^{8}$, determinó que el promedio nacional de personas con casos positivos fue de 1317,46, los departamentos y distritos de Colombia que están por encima de este referente son en su orden Bogotá (1545.8), Barranquilla (4.862), Valle del Cauca (4.690), Cartagena (4.564), Atlántico (4.215), Amazonas (2.111), Nariño (2.081), Antioquía (1.884), y Cundinamarca (1.657). De acuerdo con este estudio, se evidencia realizar una investigación por grupo etario (edades), cuyo objetivo es comparar si hay diferencias en los promedios de personas con contagio positivo y analizar qué grupo de edad es más vulnerable al contagio por el coronavirus, para ello se tomó la información registrada por el Instituto Nacional de Salud, entre los datos acumulados de los días 6 a 10 de julio de 2020.

\section{METODOLOGÍA}

El trabajo de investigación se realizó mediante un enfoque cuantitativo; la investigación cuantitativa es aquella en la que se acumulan y examinan datos cuantitativos sobre variables y estudia las propiedades y fenómenos cuantitativos. Ente las técnicas de análisis se encuentran: análisis descriptivo y exploratorio, inferencial univariable, multivariado, modelización y contrastación. ${ }^{9}$

De acuerdo con Shuttleworth, citado por Díaz ${ }^{10}$ los experimentos cuantitativos utilizan un formato estándar con algunas pequeñas diferencias interdisciplinarias para generar una hipótesis que será probada o desmentida. Esta hipótesis debe ser demostrable por medios matemáticos y estadísticos y constituye la base alrededor de la cual se diseña todo el experimento. Según Hurtado y Toro (1998) citado por Díaz ${ }^{11}$, La Investigación Cuantitativa: "La investigación cuantitativa tiene una concepción lineal, es decir que haya claridad entre los elementos que conforman el problema, que tenga definición, limitarlos y saber con exactitud cómo se inicia el problema, también es importante saber qué tipo de incidencia existe entre sus elementos".

\section{Población}

Esta investigación se centró en los diez grupos de edades con contagio, recuperadas y fallecidas por COVID-19, con la información proveniente del Instituto Nacional de Salud ${ }^{12,}$ en el periodo comprendido entre el 6 y 10 de julio 2020 (tabla 1). 
Tabla 1. Número de personas, contagiadas, recuperadas, y fallecidas por grupo etario

\begin{tabular}{|c|c|c|c|c|c|c|c|c|c|c|}
\hline Periodo & $0-9$ & $10-19$ & $20-29$ & $30-39$ & $40-49$ & $50-59$ & $60-69$ & $70-79$ & $80-89$ & $90-99$ \\
\hline $6 / 07 / 2020$ & 4827 & 8484 & 26375 & 27821 & 19152 & 15565 & 9554 & 5260 & 2652 & 591 \\
\hline $7 / 07 / 2020$ & 4599 & 8690 & 27298 & 28872 & 19912 & 16103 & 9862 & 5418 & 2762 & 606 \\
\hline $8 / 07 / 2020$ & 5124 & 8909 & 28170 & 29901 & 20589 & 16667 & 10200 & 5591 & 2860 & 627 \\
\hline 9/07/2020 & 5281 & 9220 & 29354 & 31200 & 21430 & 17385 & 10628 & 5825 & 2990 & 660 \\
\hline 10/07/2020 & 5487 & 9606 & 30873 & 32946 & 22507 & 18311 & 11126 & 6114 & 3117 & 689 \\
\hline Recuperados & $0-9$ & $10-19$ & $20-29$ & $30-39$ & $40-49$ & $50-59$ & $60-69$ & $70-79$ & $80-89$ & $90-99$ \\
\hline $6 / 07 / 2020$ & 2286 & 4069 & 11741 & 11861 & 8140 & 6340 & 3468 & 1676 & 654 & 135 \\
\hline $7 / 07 / 2020$ & 2348 & 4182 & 12083 & 12255 & 8404 & 6503 & 3566 & 1716 & 666 & 138 \\
\hline $8 / 07 / 2020$ & 2411 & 4306 & 12531 & 12712 & 8705 & 6717 & 3662 & 1752 & 697 & 141 \\
\hline $9 / 07 / 2020$ & 2494 & 4490 & 13217 & 13338 & 9158 & 7051 & 3829 & 1829 & 722 & 144 \\
\hline $10 / 07 / 2020$ & 2597 & 4669 & 13821 & 14027 & 9561 & 7355 & 3985 & 1893 & 744 & 148 \\
\hline Fallecidos & $0-9$ & $10-19$ & 20-29 & 30-39 & $40-49$ & $50-59$ & $60-69$ & 70-79 & $80-89$ & 90-99 \\
\hline $6 / 07 / 2020$ & 11 & 6 & 65 & 145 & 323 & 594 & 987 & 1034 & 817 & 228 \\
\hline $7 / 07 / 2020$ & 11 & 6 & 66 & 153 & 335 & 617 & 1019 & 1064 & 847 & 241 \\
\hline $8 / 07 / 2020$ & 11 & 6 & 66 & 158 & 345 & 644 & 1056 & 1115 & 878 & 248 \\
\hline $9 / 07 / 2020$ & 11 & 6 & 70 & 159 & 357 & 672 & 1096 & 1170 & 917 & 256 \\
\hline 10/07/2020 & 11 & 6 & 70 & 165 & 377 & 701 & 1142 & 1220 & 957 & 276 \\
\hline
\end{tabular}

Fuente: el autor

\section{Análisis estadístico}

Se plantearon hipótesis para la utilización de una prueba de comparación de medias utilizando el ANOVA para la variable contagio por COVID-19, diseñándose como una relación causal y se expone de la siguiente forma:

\section{Hipótesis con el ANOVA-Contagios}

La hipótesis del presente trabajo de investigación se diseña como una relación causal y se enuncia de la siguiente forma: Hipótesis alterna (Ha): hay diferencias en el promedio de los puntajes obtenidos por el contagio del COVID-19 entre los diferentes grupos de edades en Colombia. Hipótesis nula (Ho): no Hay diferencias en el promedio de los puntajes obtenidos por el contagio del COVID-19 entre los diferentes grupos de edades en Colombia. Prueba estadística: el valor de significancia de la prueba es de $\alpha=0.05$ (5\%), si es mayor se acepta la hipótesis nula si es menor se rechaza la hipótesis nula. Comparación entre medias independientes, se utilizó el Software SPSS v 25.0.

\section{RESULTADOS}

En la tabla 2, se observa que el p-valor de la prueba es 0,000 es menor a $\alpha=0.05$, de esta manera se rechaza Ho, es decir que para al menos uno de los promedios es diferente de los demás.
Ahora cuando Ho se rechaza en la prueba ANOVA, entonces se procede a realizar una prueba post hoc, en este caso la prueba de Duncan que es un test de comparaciones múltiples. Admite comparar las medias de los t niveles de un factor posteriormente de haber rechazado la hipótesis nula de igualdad de medias mediante la técnica ANOVA ${ }^{13}$ para muestras independientes y observar si hay diferencias entre cada uno de los niveles.

Se puede apreciar en la tabla 3, ocho subconjuntos, en ella se observan en el subconjunto 1 al grupo etario con menor promedio de personas contagiadas por el COVID-19 y así sucesivamente hasta llegar al subconjunto ocho, con el grupo etario con mayor promedio de contagio. Podemos apreciar que los grupos etarios con menor promedio de personas contagiadas son: de 90-99 $(634,6), 80-89(2.876,2)$, 0-9 $(5.063,6), 70-79(5.641,6), 10-19(8.981,8)$. Los grupos etarios con mayor promedio de personas contagiadas por el COVID-19 son: de 60-69 (10.274), 50-59 (16.806,2), de 40-49 (20.718), 20-29 (28.414), y de 30-39 (30.148).

En la figura 1 se describe las medias de contagio por grupo etario, el promedio de contagios es de 12.955,8, ahora, los grupos que están por encima de este promedio son: 30-39 (30.148); 20-29 (28.414); 40-49 (20.718); 50-59 (16.806,2), y los grupos que están por debajo del promedio de contagio

Tabla 2. ANOVA-Contagios

\begin{tabular}{|l|c|c|c|c|c|}
\hline & Suma de cuadrados & gl & Media cuadrática & F \\
\hline Entre grupos & 5008921962,000 & 9 & 556546884,667 & 513,660 \\
\hline Dentro de grupos & 43339718,000 & 40 & 1083492,950 & 0,000 \\
\hline Total & 5052261680,000 & 49 & & \\
\hline
\end{tabular}

Fuente: el autor 
son: $60-69$ (10.274); 10-19 (8.981,8); 70-79 (5.641,6); 0-9 ( $5.063,6) ; 80-89(2.876,2) ;$ y $90-99(634,6)$.

\section{Hipótesis con el ANOVA-Recuperados}

Hipótesis alterna (Ha): hay diferencias en el promedio de los puntajes obtenidos por personas recuperadas por COVID-19 entre los diferentes grupos de edades en Colombia. Hipótesis nula (Ho): no hay diferencias en el promedio de los puntajes obtenidos por personas recuperadas por COVID-19 entre los diferentes grupos de edades en Colombia.

En la tabla 4 se observa que el p-valor de la prueba es 0,000 es menor a $\alpha=0.05$, de esta manera se rechaza Ho, es decir que para al menos uno de los promedios es diferente de los demás.

Tabla 3. Comparaciones múltiples. Prueba de Duncana. Subconjuntos 1 al 8

\begin{tabular}{|c|c|c|c|c|c|c|c|c|c|}
\hline Grupo Etario & $\mathbf{N}$ & 1 & 2 & 3 & 4 & 5 & 6 & 7 & 8 \\
\hline $90-99$ & 5 & 634,6 & & & & & & & \\
\hline $80-89$ & 5 & & 2876,2 & & & & & & \\
\hline $70-79$ & 5 & & & 5063,6 & & & & & \\
\hline $0-9$ & 5 & & & 5641,6 & & & & & \\
\hline $60-69$ & 5 & & & & 8981,8 & & & & \\
\hline $10-19$ & 5 & & & & 10274,0 & & & & \\
\hline $50-59$ & 5 & & & & & 16806,2 & & & \\
\hline $40-49$ & 5 & & & & & & 20718,0 & & \\
\hline $20-29$ & 5 & & & & & & & 28414,0 & \\
\hline $30-39$ & 5 & & & & & & & & 30148,0 \\
\hline Sig. & & 1,000 & 1,000 & ,385 & ,057 & 1,000 & 1,000 & 1,000 & 1,000 \\
\hline
\end{tabular}

Se visualizan las medias para los grupos en los subconjuntos homogéneos.a. Utiliza el tamaño de la muestra de la media armónica =5,000.Fuente: el autor.

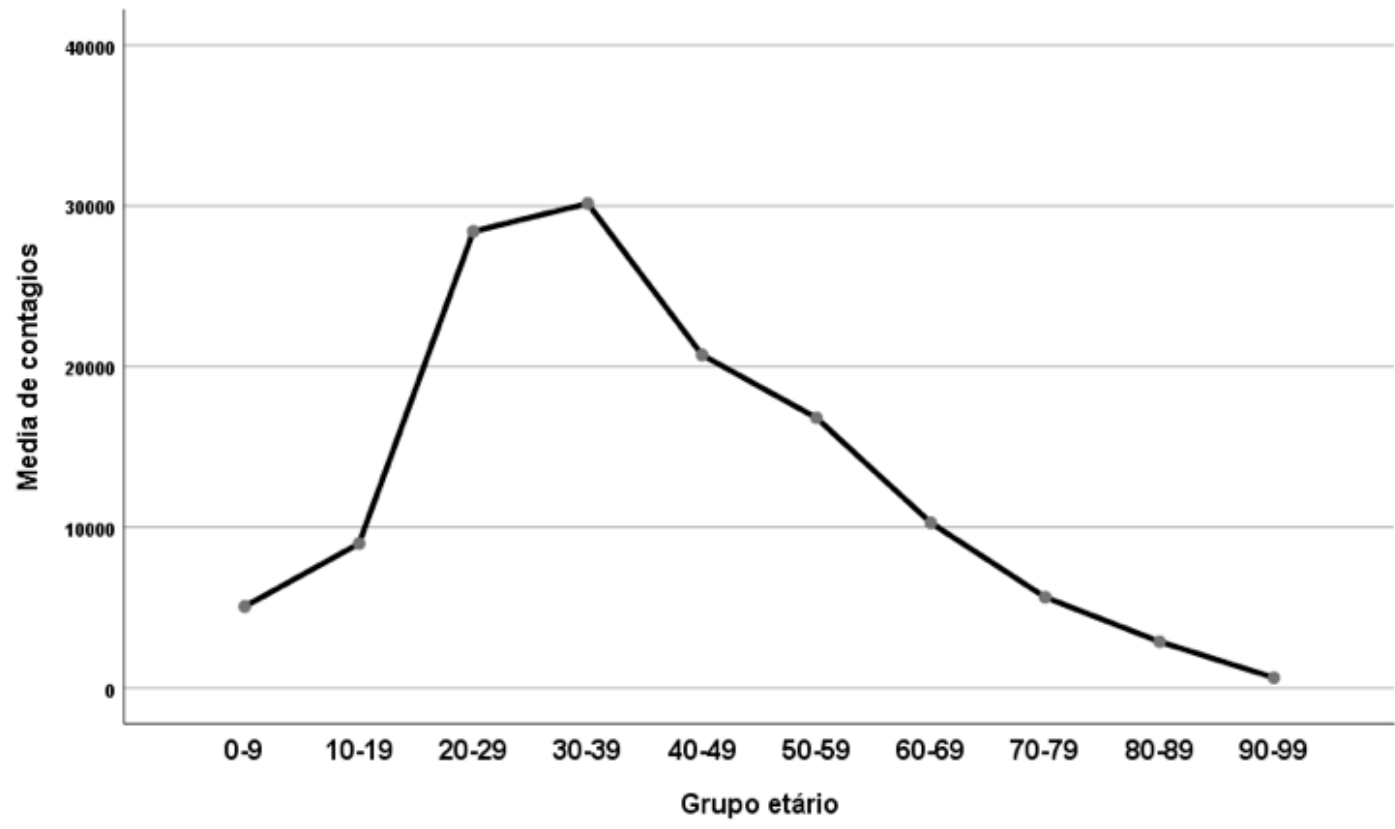

Figura 1. Medias contagio por grupo etario. Fuente: el autor

Tabla 4. ANOVA-Recuperados

\begin{tabular}{|l|c|c|c|c|c|}
\hline & Suma de euadrados & gl & Media cuadrática & F & Significancia \\
\hline Entre grupos & 987665002,820 & 9 & 109740555,869 & 528,357 & 0,000 \\
\hline Dentro de grupos & 8308066,800 & 40 & 207701,670 & & \\
\hline Total & 995973069,620 & 49 & & & \\
\hline
\end{tabular}

Fuente: el autor 
Tabla 5. Comparaciones múltiples. Prueba de Duncana. Subconjuntos 1 al 8

\begin{tabular}{|c|c|c|c|c|c|c|c|c|c|}
\hline \multirow[b]{2}{*}{ Grupo Etario } & \multicolumn{9}{|c|}{ Subconjunto para alfa $=0.05$} \\
\hline & $\mathbf{N}$ & 1 & 2 & 3 & 4 & 5 & 6 & 7 & 8 \\
\hline $90-99$ & 5 & 141,20 & & & & & & & \\
\hline $80-89$ & 5 & 696,60 & & & & & & & \\
\hline $70-79$ & 5 & & 1773,20 & & & & & & \\
\hline $0-9$ & 5 & & & 2427,20 & & & & & \\
\hline $60-69$ & 5 & & & & 3702,00 & & & & \\
\hline 10-19 & 5 & & & & & 4343,20 & & & \\
\hline $50-59$ & 5 & & & & & & 6793,20 & & \\
\hline $40-49$ & 5 & & & & & & & 8793,60 & \\
\hline $20-29$ & 5 & & & & & & & & 12678,60 \\
\hline $30-39$ & 5 & & & & & & & & 12838,60 \\
\hline Sig. & & ,061 & 1,000 & 1,000 & 1,000 & 1,000 & 1,000 & 1,000 &, 582 \\
\hline
\end{tabular}

Se visualizan las medias para los grupos en los subconjuntos homogéneos. a. Utiliza el tamaño de la muestra de la media armónica = 5,000. Fuente: el autor.

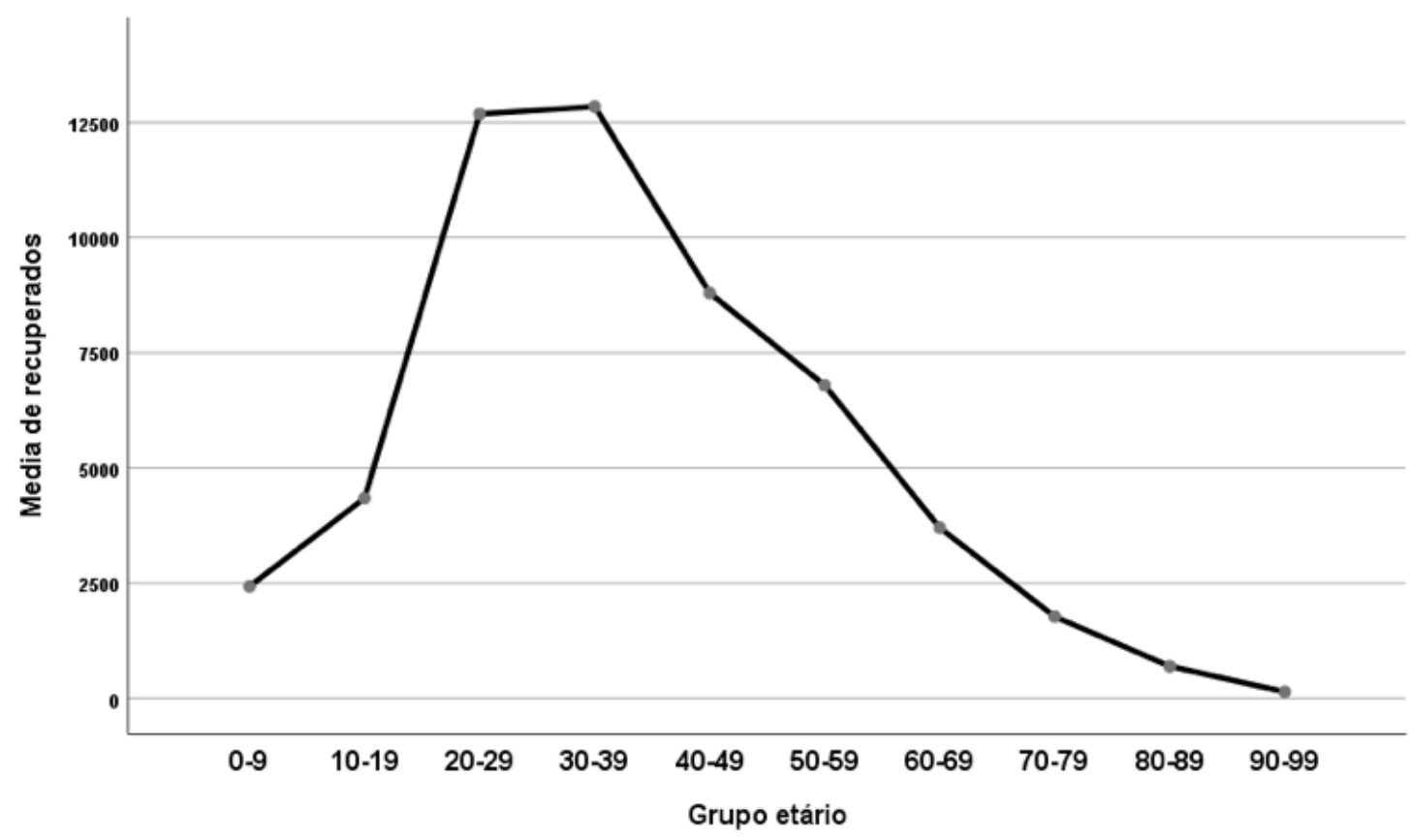

Figura 2. Medias recuperados por grupo etario. Fuente: el autor

Ahora cuando Ho se rechaza en la prueba ANOVA, entonces se procede a realizar una prueba post hoc, en este caso la prueba de Duncan que es un test de comparaciones múltiples, para el caso de personas recuperadas.

Se puede considerar en la tabla 5, ocho subconjuntos, en ella se observan en el subconjunto 1 al grupo etario con menor promedio de personas recuperadas por el COVID-19 y así sucesivamente hasta llegar al subconjunto ocho, con los grupos etarios con mayor promedio de recuperados. Podemos estimar que los grupos etarios con menor promedio de personas recuperadas son: de 90-99 $(141,2), 80-89(696,6)$,
$70-79(1.773,2), 0-9(2.427,2), 60-69(3.702,0)$, y de $10-19$ $(4.343,2)$. Los grupos etarios con mayor promedio de personas recuperadas por el COVID-19 son: de 10-19 $(4.343,2),(6.793,2)$, $40-49(8.793,6), 20-29(12.678,6)$, y de 30-39 $(12.838,6)$.

En la figura 2 se estima las medias de personas recuperadas por grupo etario, el promedio de personas recuperadas es de 5.417,8, ahora, los grupos que están por encima de este promedio son: $30-39$ (12.838,6); 20-29 (12.678,6); 40-49 $(8.793,6)$; $50-59(6.793,6)$. Ahora, los grupos que están por debajo del promedio son: 10-19 (4.343,2); 60-69 (3.702); 0-9 $(2.427,2) ; 70-79(1.773,2) ; 80-89(696,6) ;$ y $90-99(141,2)$. 


\section{Hipótesis con el ANOVA-Fallecidos}

Hipótesis alterna (Ha): hay diferencias en el promedio de los puntajes obtenidos por personas fallecidas por COVID-19 entre los diferentes grupos de edades en Colombia. Hipótesis nula (Ho): no hay diferencias en el promedio de los puntajes obtenidos por personas fallecidas por COVID-19 entre los diferentes grupos de edades en Colombia.

En la tabla 6, se observa que el p-valor de la prueba es 0,000 es menor a $\alpha=0.05$, de esta manera se rechaza Ho, es decir que para al menos uno de los promedios es diferente de los demás.
Ahora cuando Ho se rechaza en la prueba ANOVA, entonces se procede a realizar una prueba post hoc, en este caso la prueba de Duncan, que es un test de comparaciones múltiples, para el caso de personas fallecidas.

Se puede valorar en la tabla 7, nueve subconjuntos, en ella se observan en el subconjunto 1 al grupo etario con menor promedio de personas fallecidas por el COVID-19, así sucesivamente hasta llegar al subconjunto nueve, con los grupos etarios con mayor promedio de fallecidos. Podemos estimar que los grupos etarios con menor promedio de personas fallecidos son: de 10-19 (6), 0-9 (11), 20-29 (67,4),

Tabla 6. ANOVA-Fallecidos

\begin{tabular}{|l|c|c|c|c|c|}
\hline & Suma de cuadrados & gl & Media cuadrática & Fignificancia \\
\hline Entre grupos & 8603938,100 & 9 & 955993,122 & 627,803 \\
\hline Dentro de grupos & 60910,400 & 40 & 1522,760 & \\
\hline Total & 8664848,500 & 49 & & \\
\hline
\end{tabular}

Fuente: el autor

Tabla 7. Comparaciones múltiples. Prueba de Duncana. Subconjuntos 1 al 9

\begin{tabular}{|c|c|c|c|c|c|c|c|c|c|c|}
\hline \multirow[b]{2}{*}{ Grupo Etario } & \multicolumn{10}{|c|}{ Subconjunto para alfa $=0.05$} \\
\hline & $\mathbf{N}$ & 1 & 2 & 3 & 4 & 5 & 6 & 7 & 8 & 9 \\
\hline $10-19$ & 5 & 6,0 & & & & & & & & \\
\hline $0-9$ & 5 & 11,0 & & & & & & & & \\
\hline $20-29$ & 5 & & 67,4 & & & & & & & \\
\hline $30-39$ & 5 & & & 156,0 & & & & & & \\
\hline $90-99$ & 5 & & & & 249,8 & & & & & \\
\hline $40-49$ & 5 & & & & & 347,4 & & & & \\
\hline $50-59$ & 5 & & & & & & 645,6 & & & \\
\hline $80-89$ & 5 & & & & & & & 883,2 & & \\
\hline $60-69$ & 5 & & & & & & & & 1060,0 & \\
\hline $70-79$ & 5 & & & & & & & & & 1120,6 \\
\hline Sig. & & 840 & 1,000 & 1,000 & 1,000 & 1,000 & 1,000 & 1,000 & 1,000 & 1,000 \\
\hline
\end{tabular}

Se visualizan las medias para los grupos en los subconjuntos homogéneos. a. Utiliza el tamaño de la muestra de la media armónica = 5,000. Fuente: el autor.

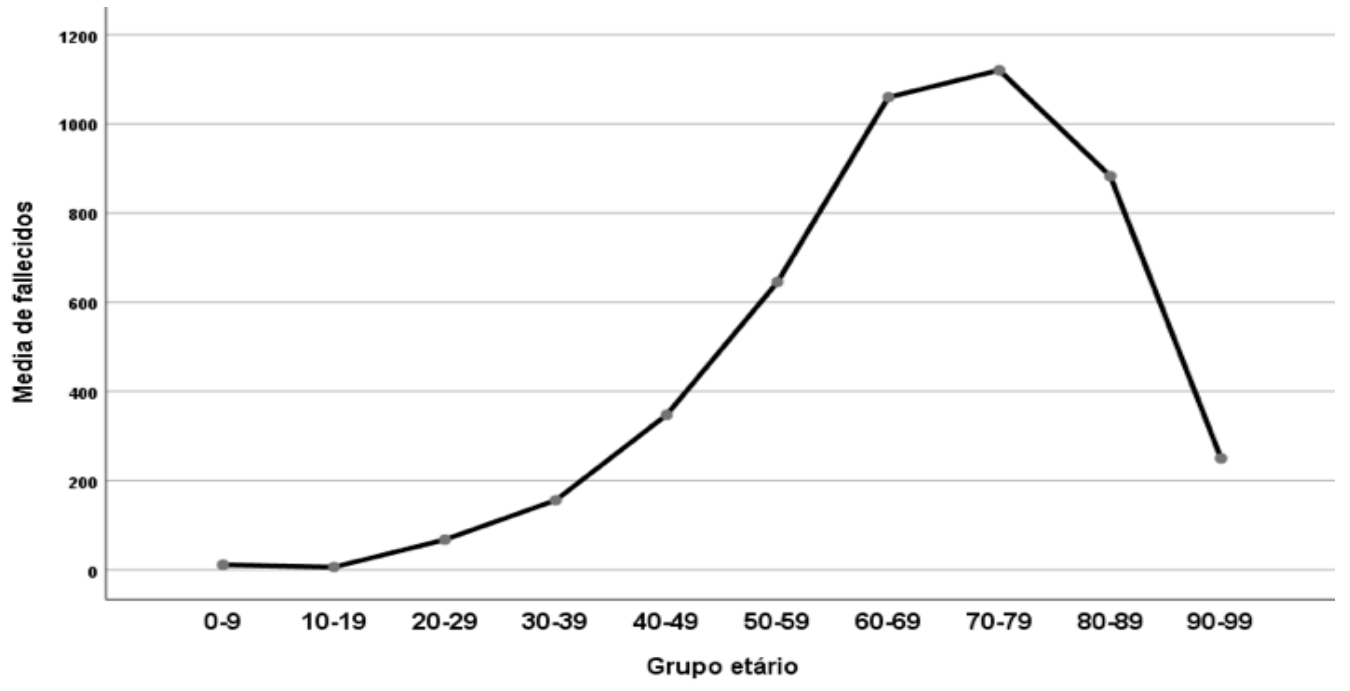

Figura 3. Medias fallecidos por grupo etario. Fuente: el autor. 
30-39 (156), 90-99 $(249,8)$, y de 40-49 $(347,4)$. Y los grupos etarios con mayor promedio de personas recuperadas por el COVID-19 son: de 50-59 (645,6), 80-89 (883,2), 60-69 (1.060), y 70-79 $(1.120,6)$.

En la figura 3 se examina las medias de personas fallecidas por grupo etario, el promedio de personas fallecidas es de 454,7, ahora, los grupos que están por encima de este promedio son: $70-79(1.120,6)$; 60-69 (1.060,0); 80-89 (883,2); $50-59(645,6)$. Ahora, los grupos que están por debajo del promedio de fallecidos son: 40-49 $(347,4)$; $90-99(249,8)$; $30-$ 39 (156,0); 20-29 (67,4); 0-9 (11,0); y 10-19 $(6,0)$.

\section{CONCLUSIONES}

A partir del análisis estadístico de los datos aplicando la comparación de medias con el ANOVA, el p-valor de las pruebas, para la variable contagios es de 0,000, este es menor a $\alpha=0.05$, de esta manera se rechaza Ho, es decir que para al menos uno de los promedios es diferente de los demás del resultado obtenido para los diferentes grupos etarios por contagio, recuperados y fallecidos de personas por COVID-19, entre el periodo comprendido entre el $6 \mathrm{y}$ 10 de julio de 2020. Se evidenció que los grupos etarios con mayor promedio de contagio fueron: 30-39 (30.148); 20-29 (28.414); 40-49 (20.718); 50-59 (16.806,2); 60-69 (10.274); y los grupos con menor promedio de contagio fueron: 1019 (8.981,8); 70-79 (5.641,6); 0-9 (5.063,6);80-89 (2.876,2); y $90-99(634,6)$. Ahora, para los grupos etarios con mayor promedio de recuperados fueron: $30-39$ (12.838,6); 20-29 $(12.678,6)$; 40-49 (8.793,6); 50-59 (6.793,6); 10-19 (4.343,2); y los grupos con menor promedio de recuperados fueron: 60-69 (3.702); 0-9 (2.427,2); 70-79 (1.773,2); 80-89 (696,6); y 90-99 $(141,2)$. Para el caso de los grupos etarios con mayor promedio de fallecidos fueron: 70-79 (1.120,6); 60-69 (1.060,0); 80-89 (883,2); 50-59 (645,6); 40-49 (347,4); y los grupos con menor promedio de recuperados fueron: 90-99 $(249,8)$; 30-39 (156,0); 20-29 (67,4); 0-9 (11,0); y 10-19 $(6,0)$.

\section{REFERENCIAS}

1. Organización Mundial de la Salud. Nombrando la enfermedad por coronavirus (COVID-19) y el virus que la causa [Internet]. [Citado 06 de julio de 2020]. Disponible en: https://www.who. int/emergencies/diseases/novel-coronavirus-2019/technical- guidance/naming-the-coronavirus-disease-(covid-2019)-and-thevirus-that-causes-it

2. Huang C, Wang Y, Li X, Ren L, Zhao J, et al. Clinical features of patients infected with 2019 novel coronavirus in Wuhan, China. Lancet. 2020;395(10223):497-506. doi: 10.1016/S01406736(20)30183-5

3. Remuzzi A, Remuzzi G. COVID-19 and Italy: what next?, The Lancet. 2020;395(10231):1225-1228. doi: 10.1016/S01406736(20)30627-9

4. Grasselli G, Pesenti A, Cecconi M. Critical Care Utilization for the COVID-19 Outbreak in Lombardy, Italy: Early Experience and Forecast During an Emergency Response. JAMA. 2020. doi:10.1001/jama.2020.4031

5. Xie, J., Tong, Z., Guan, X. et al. Critical care crisis and some recommendations during the COVID-19 epidemic in China. Intensive Care Med. 2020; 46(5):837-840. doi: 10.1007/s00134020-05979-7

6. Arabi, Y.M., Murthy, S. \& Webb, S. COVID-19: a novel coronavirus and a novel challenge for critical care. Intensive Care Med. 2020;46(5):833-836. doi: 10.1007/s00134-020-05955-1

7. Lacasa, L. Challen, R. Brooks-Pollock, E. Danon, L. A flexible method for optimising sharing of healthcare resources and demand in the context of the COVID-19 pandemic [Internet]. 2020. [Citado 06 de julio de 2020]. Disponible en: https://www.medrxiv.org/ content/10.1101/2020.03.31.20049239v3.full.pdf

8. Díaz Pinzón JE. Estudio comparativo entre el contagio durante la cuarentena obligada por el COVID-19 y el contagio durante la apertura gradual y controlada para algunos sectores de la economía en Colombia. Repert. Med. Cir. 2020;29(Supl. Núm.1):59-64. doi: 10.31260/RepertMedCir.01217372

9. UJAEN. Metodología Cuantitativa. [Internet]. [Citado 06 de julio de 2020]. Disponible en: http://www.ujaen.es/investiga/tics_tfg/ enfo_cuanti.html

10. Díaz Pinzón, J.E. Soporte técnico de simulación Phet en la enseñanza y aprendizaje de fracciones equivalentes [Internet]. 2016 [Citado 11 de julio de 2020]. Disponible en: https://ojs. uniquindio.edu.co/ojs/index.php/riuq/article/view/6.

11. Díaz Pinzón, J.E. Simulador applet descartes: como didáctica de enseñanza de la función cuadrática. INNOVA Research Journal. 2017;2(8):69-78. doi: 10.33890/innova.v2.n8.2017.256

12. Instituto Nacional de Salud de Colombia [Internet]. Coronavirus (COVID-19) en Colombia. 2020 [Citado 06 de julio de 2020]. Disponible en: https://bit.ly/2UNnOtl

13. Llopis, Jaume. Test de Duncan [Internet]. 2013 [citado 11 de julio de 2020]; Disponible en: https://jllopisperez.com/2013/01/28/testde-duncan/ 